\title{
Teresa de Manzanares de Alonso de Castillo Solórzano et Margot la ravaudeuse de Louis-Charles Fougeret de Monbron
}

\author{
Claudia RUIZ GARCÍA \\ Universidad Nacional Autónoma de México
}

\begin{abstract}
Este artículo compara dos obras: Teresa de Manzanares de Alonso de Castillo Solórzano y Margot la ravaudeuse de Louis-Charles Fougeret de Monbron. A lo largo del texto se revisan las características propias de las tradiciones picarescas española y francesa de los siglos XVII y XVIII. Se analizan los rasgos comunes, pero también las particularidades de cada uno de estos textos. Se contrasta la postura anticlerical de Fougeret de Monbron frente a la ausencia de crítica al respecto De Castillo Solórzano. Interesa ver los rasgos de cada uno de estos textos que anuncian la secularización de esta tradición.
\end{abstract}

Palabras Clave: Teresa de Manzanares, Alonso de Castillo Solórzano, Margot la remendona, Louis-Charles Fougeret de Monbron, tradición picaresca.

The article compares two works: Teresa de Manzanares by Alonso de Castillo Solórzano and Margot la ravaudeuse by Louis-Charles Fougeret de Monbron. Its central aim is to review the main characteristics of Spanish and French picaresque tradition of the XVII and XVIII centuries present in both texts. Special attention is paid to both common as well as specific traits, particularly those that announce the secularization of this tradition. Fourgeret de Monbron's anticlerical posture is thus contrasted with the absence of critique in De Castillo Solórzano.

Keywords: Teresa de Manzanares, Alonso de Castillo Solórzano, Margot La ravaudeuse, Louis-Charles Fougeret de Monbron, picaresque tradition.

Le thème de la picaresque féminine a été l'objet de multiples approches. La liste des critiques littéraires qui ont abordé sous différents angles des textes comme La pícara Justina, La hija de la Celestina, Teresa de Manzanares, La garduña de Sevilla et autres, est considérable. Il suffit de mentionner les études emblématiques d'Antonio Rey Hazas, de María Soledad Arredondo, de Regula Rohland de Langbenh, de Luc Torres, de Fernando Rodriguez Mansilla, de Cabo Aseguinolaza, ${ }^{1}$ entre autres, qui invitent le

\footnotetext{
${ }^{1}$ Il suffit de mentionner des œuvres fondamentales telles que: Antonio Rey Hazas, ed. Picaresca Femenina, Alonso Jerónimo de Salas Barbadillo y Alonso de Castillo Solórzano, Madrid, Plaza \& Janés, 1986; María Soledad Arredondo, "Pícaras. Mujeres de mal vivir en la narrativa del Siglo de Oro" Dicenda. Cuadernos de Filología Hispánica no. 11, Madrid, Edit. Complutense, 1993; Regula Rohland de Langbehn, A todas luces. El feminismo de la picaresca femenina hasta Defoe, Newark Delaware, Juan de la Cuesta, 2012;
} 
lecteur à comprendre les particularités et les richesses de ces narrations ainsi que leurs transformations.

Il est intéressant de s'arrêter sur ce dernier aspect et de le développer en proposant une approche comparative et contrastive de Teresa de Manzanares d'Alonso de Castillo Solórzano ${ }^{2}$ et Margot la Ravaudeuse de Louis-Charles Fougeret de Monbron. ${ }^{3}$ Plus d'un siècle sépare la parution de ces deux titres (1632-1748) qui se situent dans des contextes géographiques et historiques bien différents; d'un côté, l'Espagne de Philippe IV et de l'autre, la France illustrée. ${ }^{4}$ La nécessité de mettre en relief les traits communs, mais également les particularités de chacune de ces œuvres s'impose alors. La recherche du fil conducteur de ces deux œuvres nous permettra d'appréhender leur évolution.

Nous sommes confrontés à deux histoires dans lesquelles les protagonistes, conscients de leurs attributs physiques, utilisent leurs attraits pour se construire une vie meilleure et se libérer de leur condition de paria social. C'est le cas de Teresa de Manzanares lorsque, dès le chapitre II, la protagoniste évoque sa naissance et la disparition de ses parents: «Fue grandísimo el gusto que tuvo el francés con mi nacimiento e igual a él el cuidado con que me crió hasta edad de siete años. Salí con razonables alhajas de la madre naturaleza en cara y en voz. Mi viveza y promptitud de donaires prometieron a mis padres que había de ser única en el orbe y conocida por tal» (De Castillo Solórzano, 2012: 205).

Par contre, Margot, figure centrale de la nouvelle de Fougeret de Monbron, ne se présente pas à ses lecteurs de manière présomptueuse, habituellement utilisée dans de nombreux textes de la tradition picaresque féminine. Nous connaissons son physique

Luc Torres, Discours festif et parodie dans "La pícara Justina "de Francisco López de Úbeda. Lille, Thèses, 2002; Fernando Rodríguez Mansilla, ed., Picaresca femenina de Alonso de Castillo Solórzano. Teresa de Manzanares y la Garduña de Sevilla, Madrid / Frankfurt am Main, Universidad de Navarra, Iberoamericana / Vervuert, 2012; Fernando Cabo Aseguinolaza, El concepto de género y la literatura picaresca, Santiago, Universidad de Santiago de Compostela, 1992.

${ }^{2}$ Mireya Perez-Erdelyi en se réfèrant à cet auteur dit qu'il s'agit d'une figure littéraire importante car "disfrutó de considerable éxito [...] ya que después de la muerte de Salas Barbadillo (1635), se le consideraba el novelista de más renombre de la época", La pícara y la Dama. La imagen de las mujeres en las novelas picaresco-cortesanas de María de Zayas y Sotomayor y Alonso de Castillo Solórzano, Miami, Universal, 1979, p. 13.

${ }^{3} \quad{ }^{3}$ D'après Raymond Trousson, il s'agit d'un romancier mineur qui a commencé sa carrière littéraire dans la bohème de son temps et qui décrit parfaitement les joueurs, les truands, les prostituées, les filles des rues et la vie de parisiens aisés à la fortune d'origine douteuse Romans Libertins du XVIII siècle,París, Robert Laffont, 1993, p. 667.

${ }^{4}$ Fernando Cabo Aseguinolaza explique que "en realidad, hay grados muy diversos en la extensión posible del género: desde la picaresca española — en sí misma de alcance variable —, pasando por la inclusión de obras de la literatura alemana del XVII e inglesa y francesa del XVIII. Hasta llegar a abarcar cualquier producción de cualquier literatura, que tenga algún rasgo que vagamente pueda ser considerado, como la estructura episódica y el antihéroe, como protagonista. Todas las situaciones intermedias son posibles. Es probable que, de hecho, un cierto grado de relajamiento conceptual sea estrictamente necesario para el estudio comparativo de la picaresca, y tanto más cuando más abarcador sea éste, El concepto de género y la literatura picaresca, pp. 41-42. 
enchanteur par la réflexion du premier client qui la voit dans la maison close. En présence de Madame, qui dirige l'établissement, envouté par tant de beauté, il s'exclame:

\begin{abstract}
Ah, pour le coup, Florence, s'écria-t-il en jetant les yeux sur moi, voilà ce qui s'appelle du beau, du délicieux, du divin. Franchement, tu t'es surpassée aujourd'hui. Je te le dis au sérieux, Mademoiselle est adorable: oui, cent picques au dessus du portrait que tu m'en as fait. Sur mon honneur, c'est un ange. Je te parle vrai: foi de magistrat, j'en suis émerveillé [...] il faut que je la baise: je n'y saurais tenir (Fougeret de Monbron, 1993: 688).
\end{abstract}

Attentifs à certaines conventions qui régentent la nouvelle picaresque, les deux auteurs donnent la parole au personnage éponyme pour raconter son histoire et expliquer sa situation actuelle. Il nous paraît à cet égard nécessaire de s'arrêter sur la manière dont elles abordent leur généalogie. Aussi bien dans le texte en espagnol que dans le texte en français, les deux protagonistes se délectent à illustrer le mauvais sang qui les a fait naître. Teresa, par exemple, décrit ses parents sans pitié. De sa mère elle dit, "parece que la naturaleza repartió en ella con pródigas manos la fealdad" (De Castillo Solórzano, 2012: 186) et elle la présente comme une femme à la conduite légère et facile qui, sans se marier, a accepté la proposition de son amant de quitter la maison familiale et d'avoir de brèves relations avec lui. Elle l'appelle voleuse et l'accuse d'avoir quitté la maison de sa tante en emportant une bonne partie des gains; mais elle est assez astucieuse pour ne pas lui dire qu'elle a volé sa tante. Pour sa part, le père est un colporteur français, trompeur, joueur, goulu et ivrogne. Il meurt victime de ses propres excès alors que la mère se fait caresser par un homme qui lui a volé toutes ses économies et qui l'entraîne vers la mort. Devenue orpheline, Teresa survit grâce aux travaux de la toile et de l'aiguille, qui la convertissent en ravaudeuse. De même, Margot, à son début, vit de la couture et manie les fils et les tissus, car son métier de ravaudeuse lui permet d'aider ses parents dès la plus tendre enfance. Étant le produit d'une union clandestine entre un soldat et une ravaudeuse, elle raconte ses origines sur le même ton que Teresa, mais de manière bien plus crue. C'est par sa mère qu'elle a appris le métier, mais c'est parce que celle-ci était fainéante et qu'elle voulait que sa fille rapporte de l'argent au foyer. La protagoniste explique qu'elle était prédisposée favorablement à vivre de ce qu'offre la vie de la rue, parce que les ravaudeuses, de même que les vendeuses de fleurs, de boutons et de rubans, s'asseyaient sur un tonneau pour travailler, ce qui les exposait aux regards des laquais et des ouvriers. Sans aucune retenue et avec affront, elle annonce sa propension au plaisir en expliquant:

Ce fut en si bonne compagnie que j'ai pris les premières teintures de la belle éducation et du savoir-vivre, que j'ai beaucoup perfectionnés depuis, dans les différents états où je me suis trouvée. Ma parentèle m'avait transmis par le sang et par ses bons exemples un si grand penchant pour les plaisirs libidineux que je mourais d'envie de marcher sur ses traces (Fougeret de Monbron, 1993: 679-680). 
Teresa souligne qu'à la mort de son père, un hôte qui vivait dans la maison a occupé le rôle du père, ce qui l'oblige à s'en aller dormir dans la chambre de la servante. Margot pour sa part ne se gêne pas pour donner tous les détails de ce qui se passe dans sa maison. Elle explique que son père, sa mère et elle-même vivaient dans une chambre qui ne contenait que deux chaises en paille, quelques plats en terre ébréchés, une vieille armoire et un paillasson sans rideau où ils dormaient tous les trois. Cependant, Margot ne se limite pas à décrire les conditions de pauvreté extrême de sa vie, mais elle relève également les aspects les plus scabreux de son tempérament:

A mesure que je grandissais, je dormais d'un sommeil plus interrompu, et devenais plus attentive aux actions de mes compagnons de couche. Quelquefois ils se trémoussaient d'une manière si vigoureuse que l'élasticité du châlit me forçait à suivre tous les mouvements. Alors ils poussaient de gros soupirs en articulant à voix basse les mots les plus tendres que la passion suggérât. Cela me mettait dans une agitation insupportable. Un feu dévorant me consumait: j'étouffais; j'étais hors de moi-même. J'aurais volontiers battu ma mère, tant je lui enviais les délices qu'elle goûtait (680).

Et c'est à partir de ce moment que la brèche entre les deux nouvelles s'ouvre ou se referme, parfois de manière très discrète, parfois de manière plus évidente. Le prologue, autorisation d'imprimer et avertissement au lecteur, du texte espagnol se limite à avertir que le but principal de cette histoire est de divertir et de tirer des leçons de la vie agitée de Teresa de Manzanares, dont les aventures justifient le titre qui, plus tard, la fait connaître, La niña de los embustes; en effet, tout au long de l'histoire, elle emprunte diverses identités, use de ses talents de comédienne et effectue de nombreux déplacements: Madrid, Cordoue, Tolède, Alcala. L'auteur, qui conclut cette partie en soulignant le côté édifiant de cette histoire, explique au lecteur qu'il “podrá advertir los daños que se pueden prevenir para guardarse de engaños, para abstenerse de vicios, huyendo de vida tan libre ${ }^{5}$ y condición tan oscura" (De Castillo Solórzano, 2012: 182). Par contre, le texte français ne propose pas de prologue, mais dès la première ligne, la voix de la protagoniste prépare le lecteur en insistant sur la grande utilité qu'il pourra retirer de la lecture de ses mémoires. Elle explique que, si elle a exposé ouvertement les différentes facettes de son personnage tout au long de sa jeunesse, ce n'est ni par vanité ni par modestie, et elle ajoute: «Mon but principal est de mortifier, s'il se peut, l'amour-propre de celles qui ont fait leur petite fortune par des voies semblables aux miennes [la prostitution], et de donner au public un témoignage éclatant de ma reconnaissance, en avouant que je tiens tout ce que je possède de ses bienfaits et de sa générosité» (Fougeret de Monbron, 1993: 679).

Dans les deux œuvres, les personnages protagonistes sont dangereux, dans la mesure où ils décident d'user d'une des prérogatives essentielles de l'homme, l'exercice

\footnotetext{
${ }^{5}$ C'est moi qui souligne.
} 
de la liberté. ${ }^{6}$ Soledad Arredondo parle de "mujeres libres y resueltas, que viven de su físico y su ingenio" et elle ajoute qu'elles "son esencialmente embusteras e hipócritas" (1993: 25). On s'intéresse particulièrement à leur soif de liberté, illustrée par exemple par Teresa, qui tout au long du récit insiste sur le fait qu'en plus de sa beauté, elle réussit si bien dans ses différents métiers que, d'une certaine manière, elle parvient à échapper à sa condition marginale. Si, devenue orpheline, elle est obligée de travailler comme domestique dans une maison de dames âgées, elle le fait si bien et avec un tel talentqu'elle parvient facilement à changer de condition. Elle même s'étonne de ses mérites et déclare: "No era mi habilidad tan poca en materia de labor de costura y cualquiera curiosidad no la aprendiese luego que la viese hacer. Valiome esto para salir de criada de aquellas ancianas viejas y subir a que me estimasen por compañera suya" (De Castillo Solórzano, 2012: 229). Son habileté comme couturière est telle qu'elle peut très rapidement confectionner des perruques, ce qui lui attire la sympathie de femmes vaniteuses et d'"hombres que parecían calaveras con vida" (234). Mariée en quatre occasions à des maris jaloux, l'un d'entre eux meurt, victime de sa passion, tandis qu'un autre, joueur invétéré et violent, cherchera à la prostituer:

Llegó la rotura de Sarabia en el juego a tanto que comenzó a empeñarme los vestidos con que me había de lucir. Con esto no teníamos hora de paz, atreviéndoseme a ponerme las manos. Vino su desvergüenza a tales términos, que comenzó a decir que podía no ser singular en la comedia, sino admitir conversaciones de quien me quería bien, que otras alzaran las manos al cielo de tener ocasiones que yo para mayores aumentos. Finalmente, él me dio a entender que no le pesaría verme empleada en el príncipe que me pretendía, con lo cual vía abierta permisión a toda rotura y en él dispuesto sufrimiento para todo (343-344).

Le quatrième est un misérable avare, cependant, elle parvient à retirer un certain bénéfice de cette union. En effet, avec ce dernier elle aura quatre enfants ce qui pourrait se concevoir comme une réussite dans la mesure où cela l'installe dans la société, même si à la fin, elle perd toute sa fortune.

Margot, par ailleurs, recherche de manière délibérée une promotion sociale qui la sortira du monde terrible dans lequel elle est née. En effet, il ne faut pas perdre de vue que dès les premières pages, Margot affirme qu'elle souhaite être responsable de son propre destin. C'est ainsi qu'elle décide quand et avec qui elle perdra son pucelage, et à quel moment elle s'émancipera de l'autorité maternelle et des brutalités d'une mère qui la frappe avec un balai. Se sentant violentée: "[Elle] résolu[t] sur-le-champ de

\footnotetext{
${ }^{6}$ Selon Pérez-Erdelyi "cualquier deseo de independencia [de la mujer], de voluntad propia, se consideraba una aberración, un desafío a la naturaleza misma y al orden del universo, que consideraban al hombre como el ser dominante. Si la mujer no se mostraba conforme con el orden de las cosas, no se resignaba a su papel y llegaba a rebelarse, esto constituía una amenaza al matrimonio y a la familia, las instituciones básicas de la sociedad. Era necesario por consiguiente castigar a las mujeres agresivas que pretendían destruir la fibra de la sociedad", La pícara y la Dama. La imagen de las mujeres en las novelas picaresco-cortesanas de María de Zayas y Sotomayor y Alonso de Castillo Solórzano, p. 23
} 
[s]'émanciper, et d'aller tenter fortune où [elle] pourrai[t]" (Fougeret de Monbron, 1993 : 682). ${ }^{7}$ Plus tard, elle décide de travailler avec une «madame» dans une maison close et de l'abandonner quand cela lui conviendra pour se mettre à son compte. Devenue modèle d'un peintre, puis accompagnatrice de spectacles pour hommes vicieux, elle réussit, à la fin de ce parcours chaotique, à diriger son propre salon mondain.

On retrouve ce volontarisme chez Teresa, qui, à de multiples occasions, a exprimé son souhait d'échapper au cercle de la pauvreté. C'est ainsi qu'elle décide de se mettre à son compte, afin de pouvoir agir comme bon lui semble, sans être soumise et refrénée dans son champ d'action: "Yo estaba con tanto deseo de salir de la sujeción de que me determiné a casar" (De Castillo Solórzano, 2012: 242). Comme on l'a déjà vu, Teresa est fière de son habileté manuelle qui lui permet de réaliser avecsuccès des activités qui la sortent de l'indigence. Et le discours de Margot va dans le même sens; elle aussi se félicite de la qualité de son travail dans les métiers qu'elle exerce. Madame Florence, qui l'invite à travailler avec elle, lui suggère qu'il n'est pas interdit à une femme de gagner sa vie d'une forme ou d'une autre. Raymond Trousson souligne que le texte, par le biais de la voix de la proxénète, utilise «une rhétorique des gains et des bénéfices, calquée sur celle de la bourgeoisie» (1993: 671). En effet, Margot et sa protectrice parlent de leur métier en termes de bénéfices et avantages. Il s'agit pour elles d'une activité professionnelle banale, comme toutes les autres, qui exige un grand effort dans l'exercice du travail si elles veulent recevoir en retour un bon salaire et de bons résultats. Tout au long du récit, Margot souhaite mettre en évidence une situation sociale qu'elle ne peut changer. Elle est consciente que l'ordre social est inamovible. On retrouve dans le texte de Teresa cette même impression, en particulier, lorsqu'elle est sévèrement punie pour s'être moquée des chevaliers don Leonardo et don Esteban. Pour Rodriguez Mansilla, il est évident que dans cet épisode "el resorte aristocrático [...] ha estado siendo contenido a lo largo de todas las situaciones risibles de la novela. Cuando se atenta contra dignos caballeros toledanos la burla muestra su cara amarga hacia nuestra protagonista" (2012: 71).L'ordre social hiérarchisé par volonté divine ne peut être violé. Cependant, dans le texte français, on observe que l'argent est le seul moyen qui permet de s'émanciper, et le roman devient vite une «apologie d'un égoïsme vital, dans un monde où tout n'est qu'apparence et intérêt» (Trousson, 1993: 674). Ce qui n'empêche pas Margot de rajouter à son discours une violente critique sociale. Elle se proclame «martyre» et dans une longue réflexion, elle l'exprime ainsi:

En effet, qu'y a-t-il de plus insupportable que d'être obligé d'essuyer les caprices du premier venu; que de sourire à un faquin que nous méprisons dans l'âme; de caresser l'objet de l'aversion universelle; de nous prêter incessamment à des goûts aussi singuliers que monstrueux; en un mot, d'être éternellement couvertes du masque de l'artifice et de la simulation, de rire, de chanter, de boire, de nous livrer à toute sorte d'excès et de débauche, le plus souvent à contrecœur et avec une répugnance extrême?

\footnotetext{
${ }^{7}$ C'est moi qui souligne.
} 
Que ceux qui se figurent notre vie, un tissu des plaisirs et d'agréments, nous connaissent mal! Ces esclaves rampants et méprisables qui vivent à la cour des grands, qui ne s'y maintiennent que par mille bassesses honteuses, par les plus lâches complaisances et un déguisement éternel, ne souffrent pas la moitié des amertumes et des mortifications inséparables de notre état. Je ne fais pas difficulté de dire que si nos peines pouvaient nous être méritoires et nous tenir lieu de pénitence en ce monde, il n'y a guère de nous qui ne fût digne d'occuper une place dans le martyrologe, et ne pût être canonisée (Fougeret de Monbron, 1993: 691-692).

On ne peut minimiser la portée anticléricale et irrévérencieuse de ce roman, thème que l'on ne retrouve pas dans le texte espagnol, De Castillo Solorzano étant plus prudent, car il connaît le poids de la censure dans son temps. Au contraire, les critères et les mécanismes de publication en France au XVIII ${ }^{\mathrm{e}}$ siècle se flexibilisent pendant la régence de Philippe d'Orléans et, plus tard, sous Louis XV. C'est, en outre, l'époque où prolifèrent les livres interdits grâce aux circuits clandestins de distribution. En effet, un fort pourcentage de ces écrits français du XVIII ${ }^{\mathrm{e}}$ siècle exprime une violente critique de l'institution religieuse. Il est vrai que Margot raconte sa vie avec un luxe de détails surprenants et qu'elle semble se réjouir en décrivant les hommes qui bénéficient de ses services, et en détaillantl eurs perversions. C'est ainsi qu'elle nous présente tour à tour un magistrat sodomite, plusieurs membres du clergé, des hommes de la finance et des militaires ainsi qu'un baron allemand, un ambassadeur, etc. L'un d'eux est un haut dignitaire de l'église qui la désire. Il meurt tellement d'envie de satisfaire ses appétits lubriques qu'un jour il la reçoit dans son lit canonique. Elle le surnomme «dévorateur de potages d'eau bénite». Elle explique comment il adorait chaque partie de son corps qui devenait objet de culte et de sacrifice. Parmi ses assidus, elle reçoit également un franciscain, le frère Alexis, qui deviendra plus tard son proxénète et qui, ébloui par ses mérites, lui offrira un travail à l'opéra, l'endroit idéal et convoité pour faire fortune. Et pour l'aider, il lui offrira une espèce de manuel de bonnes manières qui lui permettra d'exercer avec talent la prostitution.

Il n'est pas innocent que l'auteur inclut de tels personnages dans ses mémoires, parce que, comme le fait remarquer le célèbre historien Emile Faguet, la littérature du XVIIIe siècle émane d'un groupe d'écrivains nombreux et combatifs, tourmentés par l'idée fixe que la religion chrétienne s'oppose et fait obstacle à la raison (2016: 94). Les hommes de lettres et les philosophes du siècle, parmi lesquels s'inscrivent les anti-catholiques, les naturalistes, les matérialistes, les constructeurs de la pensée antireligieuse et athée, coïncident avec cette idée et travaillent sur tous les fronts possibles pour attaquer l'institution religieuse. Le texte de Fougeret de Mombron, qui met en scène une série d'aventures obscènes avec des autorités cléricales, n'échappe pas à cette tendance. En ce sens, l'auteur s'identifie à l'attitude commune des écrivains du siècle. ${ }^{8}$ Ainsi le texte de Margot est très pragmatique, dans la mesure où elle affirme

\footnotetext{
${ }^{8}$ Dans plusieurs passages du texte, Margot met en relief les outrages de cette institution comme celui-ci : «Que ceci serve de leçon aux ecclésiastiques, et leur apprenne que les disgrâces, l'opprobre et le mépris sont
} 
que «mon livre est plus utile qu'un traité de morale» ou que «Les amis sont pure chimère» ou que «point d'argent point de suisse»" et qu' «il est bien difficile d'être honnête homme quand on est gueux» entre autres formules. Quant à Teresa de Manzanares, Rodriguez Mansilla reconnaît que "Los fragmentos moralizantes son algo abruptos, postizos o simplemente se eluden". Il indique que de toute manière, ils ne sont pas nécessaires "como elemento estructurante a la manera del Guzmán de Alfarache. [...] Se prescinde de críticas de índole social, como acostumbraba la picaresca debido a su "poética comprometida", porque simplemente, ya no hay lugar para las mismas (2012: 53).

Avec ces textes, on assiste à la transformation la plus importante du modèle picaresque, dans la mesure où, comme l'affirme Alexandre Cioranescu, les implications ou notions religieuses et morales se sont simplifiées. Le repentir et la conversion finale du héros ont disparu. L'œuvre se transforme en roman d'aventures, avec la seule différence que le protagoniste est un marginal qui peut secouer l'ordre social (1983: 508). Ils ont une préoccupation commune: progresser socialement, et peu importe le moyen. On peut affirmer que le roman s'est sécularisé et qu'il ne recherche qu'une récompense sur la terre. Dans le cas des pícaras, Eugenia Sainz González ajoute que cellesci“consiguen lo que nunca logran los pícaros: ascenso social y con él, el prestigio y la holgura económica” (1999: 34).Parfois ces pícaras volent, trompent et usurpent de fausses identités, comme ce sera le cas de Teresa qui se fait passer pour la fille d'un capitaine qui aurait disparu lorsqu'elle avait quatre ou cinq ans. ${ }^{10}$ De même, Margot usurpe, mais de manière définitive, sa place dans une société qui ne lui revient pas par naissance et lignage, car à la fin de sa vie, elle a accumulé une telle fortune qu'elle peut se retirer des affaires, et même faire venir sa mère pour profiter ensemble du bénéfice de ses rentes. Teresa qui passe par des hauts et des bas aura moins de chance. Ses aspirations

d'ordinaire la récompense de leur scandaleuse conduite. Qu'ils sachent se respecter eux-mêmes s'ils veulent être respectés. On n'est que trop convaincu que la pureté des mœurs n'est point attachée à l'habit, et que les passions ne sont pas moins vives sous la robe d'un cénobite que sous l'ajustement d'un séculier: mais on passe à l'homme du siècle ce que l'on ne passe point à l'homme d'Église: celui-ci est assujetti, à des bienséances dont l'autre est dispensé. Qu'un prêtre s'applique à sauver les apparences; qu'il sache couvrir ses vices, ses appétits, sous un extérieur vertueux et dévot; qu'il fasse sa principale étude de fasciner chrétiennement les yeux d'autrui: il a rempli ses devoirs: en exiger davantage, ce serait demander l'impossible, et contrecarrer les intentions de la nature: c'est à elle seule, et non pas à son ouvrage, qu'il appartient de faire des miracles. Que l'église donc évite de donner prise sur lui; que le vernis de la sagesse brille dans toutes ses actions extérieures; qu'il trompe, en un mot, le prochain, puisqu'il est payé pour cela; du reste, laissonsle jouir en paix». Louis-Charles Fougeret de Monbron, Margot la Ravaudeuse, pp. 722-723.

${ }^{9}$ Larivaille reconnaît que "En la vida de las putas [...] ya no predomina el sexo, sino el otro gran motor de la vida social: el dinero; El sexo se transforma en poco más que un elemento de cambio, una mercadería que la prostituta, inmune a las incitaciones eróticas, negocia y vende al mejor precio; en su lucha cotidiana por la vida, sin embargo, el antagonismo social se hace con frecuencia más evidente que la oposición de los géneros". Cité par Regula Rohland de Langbehn, El feminismo de la picaresca femenina hasta Defoe, p. 83.

${ }^{10}$ Alonso De Castillo Solórzano, Teresa de Manzanares, p. 322. "[...] antojóseme hacerme yo aquella niña robada que, según el tiempo, tendría veinte y cuatro años y de esa edad era yo". 
d'ascension sociale sont tronquées, car sa domestique lui vole tous ses biens ${ }^{11}$.Ce texte illustre la structure sociale espagnole fortement hiérarchisée, ce qui explique l'absence de dénouement heureux, bien que la protagoniste laisse espérer une suite à ce récit.

En conclusion, on peut reconnaître que ces histoires nous présentent des pícaras bourgeoises ou ennoblies, qui sont le reflet d'une société en évolution dans laquelle les règles ne sont plus figées, les valeurs commencent à évoluer et surtout la transgression ne conduit plus forcément au châtiment divin. Ce sont des romans hybrides qui évoluent entre le picaresque de cour et le picaresque galant et libertin, mais ils sont surtout très captivants et illustrent bien ce que dit avec raison Rodriguez Mansilla: "los autores epigonales parecen haber comprendido, mejor que los críticos, que no se podía [continuar reescribiendo] el Guzmán de Alfarache" (2012: 46).

\section{Obras citadas}

Arredondo, María Soledad. 1993. "Pícaras. Mujeres de mal vivir en la narrativa del Siglo de Oro". Dicenda, Cuadernos de Filología Hispánica, núm. 11. Madrid: Universidad Complutense. Pp. 11-33.

CABo Aseguinolaza, Fernando. 1992. El concepto de género y la literatura picaresca. Santiago: Universidad de Santiago de Compostela.

Castillo Solórzano, Alonso de. 2012. Teresa de Manzanares. Ed. Fernando RodRÍGUEZ MANSILLA. Universidad de Navarra, Madrid / Fráncfort del Meno: Iberoamericana / Vervuert.

Cioranescu, Alexandre. 1983. Le masque et le visage. Du baroque espagnol au classicisme français. Ginebra: Droz.

FAGUET, Emile. L'anticléricalisme. Web. 8 de abril de 2016. $<$ Obvil.paris-sorbonne.fr/corpus/critique/faguet-anticlericalisme/body-3>.

Fougeret de Monbron. 1993. Louis-Charles. Margot la ravaudeuse. Ed. Raymond Trousson. París: Robert Laffont.

Pérez-Erdelyi, Mireya. 1979. La Pícara y la Dama. La imagen de las mujeres en las novelas picaresco-cortesanas de María de Zayas y Sotomayor y Alonso de Castillo Solórzano. Miami: Universal.

Rey HazAs, Antonio, ed. 1986. Picaresca Femenina, Alonso Jerónimo de Salas Barbadillo y Alonso de Castillo Solórzano. Madrid: Plaza \& Janés.

\footnotetext{
${ }^{11}$ Il est intéressant de remarquer comment se présente le châtiment exemplaire, parce que Teresa ne reçoit pas de sanction de la justice divine, mais des hommes. En effet, bien qu'elle obtienne une fortune en lien avec certains de ses maris, elle ne peut jamais en profiter, parce qu'en général ils sont avares, ou bien ne reçoit rien de ce qu'elle avait prévu au veuvage; ou la perd grâce à la malice d'une autre.
} 
Rodríguez Mansilla, Fernando, ed. 2012. Picaresca femenina de Alonso de Castillo Solórzano. Teresa de Manzanares y la Garduna de Sevilla. Madrid / Fráncfort del Meno, Universidad de Navarra / Iberoamericana / Vervuert.

Rohland DE LANGBeHn, Regula. 2012. A todas luces. El feminismo de la picaresca femenina hasta Defoe. Newark: Juan de la Cuesta.

SAINZ GonZÁLEZ, Eugenia. 1999. "Misoginia o miedo en la picaresca femenina". Verba hispanica: Anuario del Departamento de la Lengua y Literatura Españolas de la Facultad de Filosofia y Letras de la Universidad de Ljubljana, núm. 8. Ljubljana: Univesidad de Ljubljana, 1999. [Consultado el 9 de enero de 2016.]

TORRES, Luc. 2002. Discours festif et parodie dans "La picara Justina" de Francisco López de Úbeda. Lille. Thèses. (Tesis inédita.)

Trousson, Raymond, ed. 1993. Roman libertins du XVIII siècle. París: Robert Laffont. 